\title{
AS BAD AS THINGS ARE FOR RADIO ASTRONOMY IN THE PROTECTED BANDS - - THEY ARE WORSE FOR SETI !
}

\author{
JILL TARTER \\ The SETI Institute, and Astronomy Department, UC Berkeley, Berkeley, \\ CA U.S.A.
}

\begin{abstract}
If the funding that has been requested in NASA's FY89 budget is forthcoming, the Search for Extraterrestrial Intelligence (SETI) will inaugurate its microwave search program on Columbus Day, 1992. This systematic search for evidence of narrowband microwave signals generated by another technology will continue at several sites around the world until at least 1998. The search will be conducted in two modes: a sensitive targeted search of selected solar type stars covering 1 to $3 \mathrm{GHz}$, and an all sky survey that covers 1 to $10 \mathrm{GHz}$. This region of the spectrum is the quietest in terms of natural background noise from astrophysical sources and/or the terrestrial atmosphere, which is why it was chosen.

Unfortunately, at the same time that terrestrial technology has enabled this systematic search, it also threatens to render the search all but impossible from the surface of the Earth. Terrestrial transmissions generated for an enormous number of different purposes will be the signals most frequently detected by the SETI systems! This paper describes the design decisions and observing plans that the SETI Program is now making in order to have a chance of successfully "listening" through this heavily utilized portion of the spectrum. We do not yet know precisely how heroic our efforts to discriminate against and identify RFI may have to be, but we have been accumulating observational experience and data that demonstrate convincingly that the situation is rapidly growing worse. Because of the real-time nature of the signal processing being planned, SETI systems will provide an excellent opportunity to monitor, and perhaps identify, sources of narrowband interference within the radio astronomy bands at each site where observations are being conducted.
\end{abstract}

\section{INTRODUCTION}

At the 1959 WARC there were representatives from around the world, but none from other solar systems. Extraterrestrials will thus be ignorant of the few precious bands of the frequency spectrum that radio astronomers managed to secure for the practice of their passive service. The optimal frequencies for transmission by an extraterrestrial engineer will probably be determined by the intended purpose of the transmission, the natural background radiation, achievable signal-to-noise ratios, economies based upon the judicious use of some precious resource, and sociopolitical constraints that we cannot begin to predict. While considerations of the natural astrophysical background radiation 
at various frequencies and achievable signal-to-noise ratios from known transmitter technologies can be used to argue that intentional communication over long distances might well be carried out at microwave frequencies, more cannot be said with any certainty. Thus a systematic search for evidence of another technology must not be limited to the protected radio astronomy bands, but must explore as much of the quiet microwave window as is feasible. In the near future, with realistic projections of available funding, SETI will be limited to ground based searching and thus be constrained by atmospheric noise to the frequency region from roughly 1 to $10 \mathrm{GHz}$. Years of experience at radio astronomy observatories have shown us that even within those protected, exclusive bands that astronomers use, where transmitters should not be, there is a real problem with interference. In spanning the 1 to $10 \mathrm{GHz}$ band, SETI will be operating at frequencies where terrestrial and space based transmitters are supposed to operate. We have little data concerning just how bad the interference problem will be; we know only that it will be worse than those examples, within the radio astronomy bands, that we do have.

In making plans for a ten year observational program, NASA has been attempting to design a flexible signal processing system whose capabilities can be incrementally enhanced to handle RFI. This enhancement is necessarily expensive, and will only be undertaken if and when observational field experience with a series of prototype instruments demonstrates that more extensive measures must be employed to deal with the existing and projected levels of RFI.

There is another reason to state that RFI will be a bigger problem for SETI than for radio astronomers: the nature of the signal. Radio astronomical systems are optimized for detecting weak, incoherent signals that occur over a range of contiguous frequencies - the kind of signal nature always produces. In SETI, the decision was made early to define as potentially interesting, those types of signals that, to our knowledge, nature never produces. Specifically, SETI will search for signals that are concentrated in frequency (for example, continuous wave or $\mathrm{CW}$ signals). The signals may also be concentrated in time (as in the case of regularly pulsed signals), but such signals must also be narrowband, being no broader in frequency than their duration demands. The SETI signal processing system has been optimized for the detection of such artificial signals. Therefore it should not be surprising that these detectors are far more susceptible to interference from the coherent components of artificial signals produced by terrestrial technology than are the radio astronomy detectors. The SETI systems will also be bothered by the spread spectrum interference from the GLONASS satellites that have been so often cited at this meeting. In addition, SETI will see all the individual carriers from GLONASS and each of their successive manifestations within the $\operatorname{Sin}(\mathrm{x}) / \mathrm{x}$ nulls.

\section{THE SETI OBSERVING PROGRAM}

From the Columbus Day turn-on until at least October 1, 1998, the SETI program will be conducted in two simultaneous and complementary modes at several sites around the world. There will be a Targeted Search that will concentrate on about 1000 nearby solar-type stars where we believe that there is a greater a priori probability of finding another technological civilization. This 
will be the most sensitive search mode, using the world's largest radio telescopes to observe over the 1 to $3 \mathrm{GHz}$ portion of the terrestrial microwave window where the natural radiation background is an absolute minimum. Observations at any frequency band will last for as long as $\mathbf{1 0 0 0}$ seconds. The observations will be carried out with dual circularly polarized receivers and direct Fourier transform spectrometers providing instantaneous resolutions of $1,2,4,8,16,32$ $\mathrm{Hz}$ and $1 \mathrm{KHz}$ simultaneously. These individual resolutions will be oversampled to avoid aliasing, and the sampling time will be extended, relative to the sampling time needed for the nominal resolution, so that after the application of a Hanning function the equivalent noise bandwidths of the channels will equal the values of the resolutions quoted above. Thus there will be $70 \mathrm{~dB}$ of isolation against strong signals that are just 10 channels away. In addition, there will be two spectra calculated at each resolution, with a $50 \%$ overlap in time to insure that the "scalloping" in the time domain is minimized for pulsed signals that are not synchronized with the sampling times. Pulses with repetition periods in the range $\mathbf{3 0}$ milliseconds to nearly 1000 seconds should be detectable. However, the power contained in the individual pulses of slow rep-rate signals will have to be higher in order to allow them to be detected with the same degree of statistical significance. Since the input bandwidth to the spectrometer will be at least $10 \mathrm{MHz}$, there will be in excess of $10^{7}$ channels of spectral data every time frame. The frequency-time plane will be searched for evidence of $\mathrm{CW}$ or regularly spaced narrowband pulses. These signals may be stationary in frequency or drift at rates up to $\dot{v} / \nu=10^{-9} / \mathrm{sec}$. Such a drift may occur due to relative acceleration between the transmitter and receiver. The maximum allowed drift rate has been set to correspond to the most rapid planetary rotation within our own solar system. Depending upon the size of the telescope utilized and the length of an observation, the sensitivity of the Targeted Search (in the absence of interference) will range from $10^{-26}$ to $10^{-25} \mathrm{~W} / \mathrm{m}^{2}$.

It is possible that the most detectable SETI signal may originate from the vicinity of some distant star that is too faint to appear in current stellar catalogs. As with the night sky, this signal may appear to be the brightest, not because it is the closest, but because it is intrinsically bright. The Targeted Search is likely to miss this signal because there will be no way of selecting the correct direction in advance. NASA plans to conduct a Sky Survey in parallel with the Targeted Search. The Sky Survey is likely to miss this signal because there will be no way of selecting the correct direction in advance. NASA plans to conduct a Sky Survey in parallel with the Targeted Search. The Sky Survey will not make any assumptions about which directions to select; it will search them all by scanning one of the $34 \mathrm{~m}$ antennas at one of the DSN complexes at a rate of 0.2 degrees/second for 8 hours each day. The Sky Survey will extend the frequency coverage to $10 \mathrm{GHz}$ because in the search for stronger signals it can tolerate the increased atmospheric noise above $3 \mathrm{GHz}$. The rapid scan rate permits the Sky Survey to be completed within the lifetime of the project, but limits the time spent observing any half power beamwidth on the sky at any frequency band to 3 seconds at the lowest frequency and 0.3 seconds at the highest. Because of this short dwell time, the Sky Survey will not perform the detailed type of frequencytime plane pattern recognition that is used in the Targeted Search. Instead, the Sky Survey will concentrate on detecting strong CW signals. Suspected ETI signals will be elaborately processed, taking into account the signal's behavior as the telescope moves along an individual scan line or returns to the same portion 
of the sky on an adjacent scan line. The instantaneous bandwidth of the Sky Survey signal processing system will be in excess of $250 \mathrm{MHz}$ and the final resolution of the FFT spectrometer will be $20 \mathrm{~Hz}$, so this system will also have more than $10^{7}$ channels of data to process each time frame. Dual circularly polarized, low-noise receivers will be used and (in the absence of interference) a sensitivity of between 1 and $3 \times 10^{-23} \mathrm{~W} / \mathrm{m}^{2}$ will be achieved in this mode. System sensitivity may be improved by slowing down while crossing those portions of the sky containing the galactic plane, where most of the distant stars are located.

\section{RFI SOLUTIONS}

In summarizing the achievable sensitivity of both the Targeted Search and the Sky Survey, it was necessary to include the caveat "in the absence of interference."

However, it is clear that at least at some sites and in some frequency bands, interference will be present. What options are available for dealing with RFI? All signal detection algorithms in the Sky Survey and the Targeted Search operate with some sort of threshold. That is, individual data samples or short accumulations of data will only be considered for additional processing if they exceed some threshold. If interference becomes a problem, this threshold can be automatically or manually raised until the amount of data passing the threshold drops to a level that the signal processors can continue to handle in real-time. Historically this is the way almost all SETI programs have functioned. But if the interference is very strong or very prolific, this technique can result in such a severe loss of sensitivity that it probably doesn't make any sense to continue the observation. There are other options that should be considered; they are discussed below. When and where they will be required cannot now be predicted.

\section{A. Baseline System}

Figure 1 shows a block diagram of an automated SETI system that will be usable at quiet sites in quiet bands. The system consists of low-noise, dual polarization receivers, two multichannel spectrum analyzers (MCSAs) followed by a signal recognition system consisting of a CW detector and RFI discriminator for the Sky Survey or a pulse detector and a CW detector that work on the individual polarized data streams as well as their union in the case of the Targeted Search. A control computer automatically oversees the signal processing, performing automatic error checking and self diagnostics on the hardware, as well as providing a user interface, access to an RFI database, and passing instructions to the observatory control computer. Although an observer will be on site to continue signal recognition tasks when the system cannot make a decision, this system is supposed to operate in a fully automated mode, including performing routine reobservation of detected signals that cannot be classified as RFI.

Prior to any observations at a given site, this system should be used to make an interference survey of the site. The results should be stored in rapid access databases capable of being queried in real-time for the Targeted Search and at the end of a prescribed number of scans in the Sky Survey. Candidate signals that cannot be identified by comparison with the database will require 
reobservation so false alarms will not waste precious telescope time. Any new occurrences of interference should be added to the database. As observations continue at a given site, experience with the schedules of recurring sources of interference and a frequency agility within the SETI systems may allow for some form of intelligent search scheduling that can work around the vast majority of the known sources.

This is the mode in which the Sky Survey will be operated: trading off geographic diversity and scheduling flexibility against the degradation of system sensitivity. For the Targeted Search, this mode may prove adequate most of the time. However, where this modality is inadequate, the extended time on target (relative to the Sky Survey) and the real-time signal processing used to detect drifting CW and pulses both offer other alternatives that can perhaps maintain the sensitivity of the search even in the presence of strong RFI.

\section{B. RFI Subsystem}

Much of the interference suffered by the SETI systems will enter the detectors via the side lobes of the antenna. This interference lies preferentially on the horizon and may be discriminated against by use of an anti-coincidence RFT subsystem. A small antenna having a beam pattern as closely matched as possible to the main antenna side lobe pattern, is operated in parallel with the main SETI system. Any signal that is seen simultaneously in the RFI system and the main system is considered to be interference and is excluded from further consideration. Although this is a simple concept, the signal processing implications are severe and the added costs are substantial. There is a small added cost for the RFI antenna and receivers, but the real cost impact stems from the fact that for both systems to "see" the same signal, both systems must possess identical signal processing equipment. That means that the MCSAs and signal detectors must be duplicated and the logic needed to make the comparison of signals from the two antennas must be incorporated. At this time it is believed that a single unpolarized output from the RFI receiver is sufficient. Figure 2 shows a block diagram of the system currently planned for this implementation. Note that it contains 3 MCSAs instead of 2 and the pulse detector and CW detector contain additional logic for signal comparison. An example of the comparison logic is shown in Figure 3. Individual thresholded samples of data from all resolutions are compared in the pulse detector. At a particular resolution, if the same signal is found in the RFI data stream and one or both of the two polarized data streams from the main antenna, the data are discarded and not passed along for further processing. Comparison for $\mathrm{CW}$ signals takes place only after a short stage of incoherent addition along all possible drift tracks (at either the $1 \mathrm{~Hz}$ or the $2 \mathrm{~Hz}$ resolution, depending on the frequency) for data streams from both subsystems. Tracks that are determined to be statistically significant in the data from the RFI subsystem are pruned out of the data in the main system before those data are passed along for further processing.

As an additional precaution against processor overload due to strong or numerous RFI signals, the number of samples exceeding threshold within any kilohertz resolution element are counted. If that number gets too high, that entire block of data is pushed to the end of the processing queue, and is only analyzed if the processor finishes all of the other kilohertz channels with sufficient time to spare. 
This system will not help to discriminate against weak RFI that enters in or near the main beam, such as satellite and airborne transmitters. There is a certain amount of recovery of frequencies and targets, blocked by satellites, that can be accomplished by means of rescheduling to a different site or working at a different time.

\section{Dual-Beam System}

At sites and in frequency bands where main lobe interference dominates (due to satellites, etc.) a dual beam system should be more successful in preserving sensitivity. There are additional costs beyond those already accounted for by the RFI subsystem. A second set of polarized feeds and receivers must be instrumented on the main telescope. To the greatest extent possible, the sidelobe patterns of these two RF systems should be made to match when installed on the telescope. With augmented logic similar to that used in the RFI system, the data from the two polarizations of the two beams are compared. Signals seen in data from the "off source" beam as well as in data from the beam containing the target are discarded and not subjected to further processing. Once again there is the same ability to eliminate entire kilohertz bands from further consideration by the signal processors in order to avoid overloading those processors. The additional costs for the second beam are modest. The major costs result from the addition of another MSCA and the implementation of the required logic along the data paths shown in Figure 4.

\section{Multiplying Interferometer}

At some sites and in some bands the loss in sensitivity and frequency coverage suffered when many kilohertz worth of spectral data went unprocessed, rending the observations useless. If more than one telescope exists and can be scheduled at a site, there is the possibility for implementing a multiplying interferometer. Such an instrument provides a matched filter for a point source moving on the sky at sidereal rate. Signals from all other sources are automatically discriminated against. Thompson (1982) at the VLA has demonstrated just how well such an instrument suppresses interference. Welch (1986) has shown what benefits SETT can expect from an interferometer. For a $1 \mathrm{Km}$ baseline and a 30 second coherent integration, the fringe rate suppression factor for an interfering signal is nearly 100 . This benefit can be exploited in the short accumulation stage used by the CW detector. it is less obvious, but the multiplying interferometer can offer RFI protection even for signal processing algorithms based on individual data samples. If the baseline is extended to just over $4 \mathrm{Km}$, a coherent terrestrial transmitter at $1 \mathrm{GHz}$ will be shifted by one $1 \mathrm{~Hz}$ channel in the output from the MCSAs on one antenna compared to the output of the MCSAs on the other antenna. Although such frequency shifted data samples increase the overall noise, they cannot add coherently along any drift path. For individual pulses, one must look at the phase of the thresholded samples. Individual pulses coming from the target direction on the sky will all have a constant phase (usually set to zero); pulses arriving from another location will have a variable phase. In the pulse detection algorithm, every possible pair of thresholded data points is formed and then a search is conducted for a third, above-threshold point that could represent a continuation of the pulse train. In the presence of interference, many more data samples will exceed a given threshold, thus increasing the number of initial pairs and taxing the 
computational capability of the Pulse Detector during the search for a third pulse. By automatically disqualifying any pairs of thresholded data that do not have identical phase values, the number of qualifying pairs is reduced and the processor can be protected from overload.

The incremental costs to implement this observational mode are non-trivial. As with the dual beam system, two sets of feeds and receivers must be supplied. A delay line and fringe rotator must be introduced into the data path from one of the antennas (if it does not already exist at a particular site). A capability to perform a channel-by-channel complex multiply on the MCSA outputs from the polarized data streams from each antenna, as well as on the union of the polarized data, has to be inserted. Finally, all of the signal processing hardware must be able to perform complex arithmetic and accumulation. Figure 5 illustrates the kind of system that is needed.

Clearly this type of system is the most complex, but wherever two or more antennas of comparable size exist, this mode of observing offers the greatest protection against interference with the least sacrifice in sensitivity. One thing that should be obvious from Figure 5 is that, with the exception of the added delay line, the complex multiplier and increased computational capacity and memory to deal with complex arithmetic, this system looks like two side-by-side examples of the baseline system. Therein lies the key to SETI planning for RFI. If it turns out that initially the RFI environment is unknown or gives the false impression of appearing relatively benign, it is still possible to proceed with the construction of the required number of $10 \mathrm{MHz}$ baseline systems for the Targeted Search (6 are now planned) and adapt them at a later date, if necessary, so long as the interfaces and processors are designed to accommodate the enhancements needed for the incremental systems. In the event that conditions dictate the use of an RFI subsystem, a dual beam system, or a multiplying interferometer at some sites and at some frequencies, existing baseline systems can be enhanced and additional baseline systems and the enhancements can be procured and added. If fiscal constraints preclude the production of additional baseline systems, one could choose to move existing systems around and schedule time so that the original systems can be paired together and enhanced with the necessary additional hardware in order to create one or more interferometers as needed.

\section{E. Temporal Accommodation}

RFI may be so prevalent that at some sites and in some bands, none of the above implementations of SETI systems allow a search to be conducted to meaningful sensitivity levels. There is another possible option open to SETI with which radio astronomy has had some small historical success. It may be possible to identify and then contact the authorities controlling at least a subset of the worst sources of interference. Since at any site the number of targets, number of frequency bands and the total time required to observe the hitherto obscured frequencies are countable and finite, it may be possible to arrange to have transmitters turned off on some schedule that will permit the completion of the SETI search. The cost of this modality is primarily the cost of conducting the identification procedures and the political negotiations. Whether it is really an option will depend on just how many $10 \mathrm{MHz}$ bands are corrupted beyond repair by any of the above schemes and how many sources of interference are blocking each band. 


\section{F. Far Above the Earth}

To search through the upper end of the free space microwave window (to about $100 \mathrm{GHz}$ ) requires access to space above the noise of the Earth's atmosphere. RFI may eventually dictate this same access to space even for frequencies at the low end of the window. Detectors that are below the geosynchronous orbit suffer strong interference from the satellites in that orbit. Therefore future SETI systems will have to be launched into some high orbit above the geosynchronous satellites and carry with them a shield that is deployed in the Earth pointing direction. In addition, one can take advantage of the natural shielding offered by the moon and conduct a SETI search from the lunar farside. The costs of either alternative are huge. An old engineering study by Basler et al (1976) concluded that the moon offered a more cost effective opportunity.

This choice can only be properly revisited at such time as the country makes a decision about what its long term goals for space exploration will be. For the time being, the NASA SETI Program will concentrate on a ground based search through the terrestrial microwave window and attempt to manage the RFI problem with the evolutionary approach to hardware systems listed above.

\section{TOOLS FOR EXPLORING THE RFI ENVIRONMENT}

It should be obvious from the foregoing discussion that a quantitative description of the current and projected RFI environment (throughout the 1 to $10 \mathrm{GHz}$ frequency range) is absolutely necessary in order to make reasonable decisions on hardware production for the Targeted Search and on the observing strategies and signal recognition algorithms for the Sky Survey. Some rudimentary tools to help investigate this question exist today, and improved versions will be available in the next year or two.

\section{A. Existing Tools}

As described by Sam Gulkis in another paper during this meeting, JPL has built an RFI SETI Surveillance System and used it to study the 1 to $10 \mathrm{GHz}$ environment at the Goldstone DSN site. The general results seem to be that at the far outside lobe level of a high gain antenna, $1 \%$ of the frequency range is obscured $10 \%$ of the time. The most heavily obscured frequencies (20\%) are 1 to $2 \mathrm{GHz}$. Increasing the sensitivity of the search does not raise the number of interference events as rapidly as predicted by a uniformly distributed population of interfering sources. It appears that most of the sources are contained within the DSN complex and the neighboring Army base. The mountains appear to provide good shielding against more distant terrestrial sources of interference. It is hoped that funds will be available during FY89 and 90 to permit the RSSS to be deployed and operated at a number of observatories and sites around the world which are currently being considered for use in the SETI Program. A comparative survey of the current levels of interference could probably be completed with a few months at each site. The sensitivity levels achieved by this approach will not address the question of interference that enters in or near the main telescope beam, but the relative results should be most useful.

To test out the proposed digital architecture for the Targeted Search MCSA, a wirewrap prototype was built by Stanford University (Linscott, Chen 
and Peterson, 1985) and tested at the Venus station in the Goldstone Complex. This prototype has 74000 spectral channels with resolutions of $0.47 \mathrm{~Hz}$. It is controlled by a VAX 11/750 that also serves as a host for the real-time signal detection algorithms, and it uses a SUN 100 graphics workstation for display purposes. Pulse detection algorithms can keep up with the full bandwidth of the data, but $\mathrm{CW}$ algorithms are too computationally intensive and can manage only about 4000 channels of data in real-time. For the purposes of subsequent offline analysis, about 40000 channels of data can be recorded to disk continuously until the disk is filled. A large number of "spurs" were discovered in the LO chain at this site. The limited bandwidth of the system and the limited access to receivers and feeds beyond $\mathrm{S}$ and $\mathrm{X}$ band made it difficult to use this prototype by itself for interference surveys over the 1 to $10 \mathrm{GHz}$ region. However, if used in conjunction with the RSSS, this system can provide a high sensitivity analysis of individual regions that the RSSS identifies as particularly obscured. At the end of 1987 this system was shipped to Arecibo in hopes of using it to do some RFI studies as well as conducting several astronomical observations. The instrument was damaged in transit and has not yet been repaired. We look forward to its recovery and future use in conjunction with the RSSS at Arecibo. Shipment to another site is probably not feasible in the near future.

The Nancay Observatory has constructed a 3-bit, 8-level autocorrelator for use in spectral line observations. Since the early 1980 s this system has been used to provide 1024 channels, with a resolution of $50 \mathrm{~Hz}$, in order to conduct a SETI search of solar-type targets that lie beyond the declination range of Arecibo. Each target is tracked for $1 / 2$ hour and the LO is stepped to cover $0.64 \mathrm{MHz}$ around the $\mathrm{HI}$ line and $2.2 \mathrm{MHz}$ around the $18 \mathrm{~cm} \mathrm{OH}$ main lines. A week of observing produces 6 or 7 million channels worth of spectral data characterized by a correlator integration time of 30 seconds. This is almost equivalent to the data set corresponding to a short 30 second accumulation for $\mathrm{CW}$ signals along 1 drift path in the frequency-time plane, for one polarization with one $10 \mathrm{MHz}$ Targeted Search MCSA. It has been possible to make a study of the number of events occurring as a function of the accumulated power normalized by the expected accumulated mean power per channel. Figure 6 presents the results for the last 5 observing sessions at Nancay. The data are scaled to the extrapolated number of events expected within 10 million channels from a single polarization MCSA and they are compared to the theoretical predictions from Gaussian noise alone. At normalized powers close to 1.1, there is an "excess" noise whose behavior parallels the Gaussian prediction. This is probably due to a bias introduced in the estimation of and normalization by $P_{\text {mean }}$ as well as a large number of weak discrete digital signals indigenous to the observatory environment. A very slight increase in an applied threshold in this region will result in a substantial decrease in the number of events without much loss in sensitivity. At larger values of normalized power there is clearly a second population of signals which is produced by individual incidences of interference. The great majority of these events occurred at frequencies near the $21 \mathrm{~cm}$ line, an exclusive band for radio astronomy. The question is whether or not in other bands the rate of occurrence raises this curve so high that at false alarm probabilities (noise alone) of $10^{-2}$ to $10^{-3}$ these events dominate the statistics of the"excess" noise. Since the slope of these curves is shallow, a very large increase in threshold would be required to bring the event level down to a value that the processors could cope with. At any new site, we can expect that such curves of 
interfering events will be the norm. Deliberate attempts will have to be made to identify the offending signals and excise any that are within the observatory and accessible. External sources of interference will have to be handled with one of the RFI solutions presented above.

\section{B. Near Term Interference Monitors}

The sensitivity of RSSS is limited and the instantaneous frequency coverage of the other two systems mentioned above is very small. It is unfortunately true that one must build something close to the final SETI systems in order to survey the RFI environment meaningfully. Two more prototypes will be completed in the near future that are adequate to this task.

The DSN is constructing a wideband spectrum analyzer for use in spacecraft tracking. The system will have an input bandwidth of $40 \mathrm{MHz}$ single polarization or $20 \mathrm{MHz}$ dual polarization. Since this represents a substantial fraction of the eventual $250 \mathrm{MHz}$ bandwidth of the Sky Survey system, JPL has taken advantage of this opportunity to implement an engineering design model (EDM). The DSN has agreed to provide a $20 \mathrm{~Hz}$ output resolution and JPL is producing prototypes of the signal processing and pattern resolution hardware and software that will be implemented in the final Sky Survey system. In this way, a $40 \mathrm{MHz}$ prototype of the Sky Survey system should be available by the end of 1990. Since it makes partial use of DSN equipment, it is unlikely that it will be able to be removed from Goldstone, but at least at that site the EDM should permit a reasonable RFI study to meaningful sensitivity levels. Figure 7 illustrates the major components of the EDM.A second generation Targeted Search Prototype (TSP) is currently under construction. This system is based upon a custom VLSI fast Fourier transform signal processing chip designed by Stanford University (Duluk et al, 1986). Signal processing and system control are managed by a MicroVAX and the display system is a SUN 3/260 workstation. In addition to verifying the chip and system designs, this prototype will offer all the simultaneous resolutions, full bandwidth at one polarization or half bandwidth at dual polarization, and the opportunity to experiment with "smart" schedulers and databases. The MicroVAX will handle the pulse detection in real-time, but the implemented real-time $\mathrm{CW}$ algorithms will not approach the optimal level of sensitivity. In the future it is expected that commercially available parallel processing computer architectures will be able to handle the CW signal detection algorithms at the full data rate and full sensitivity. The TSP also offers the first real opportunity to do RFI studies over the full range of frequencies (if a suitable inexpensive feed/receiver system can be procured). The first deployment of the system will not be until 1990, but it will afford the opportunity to develop databases at observatory sites before the observing actually begins in 1992. RFI experience will be incorporated into the procurement process for the final six Targeted Search systems, as it becomes available. Figure 8 shows the configuration of the TSP when it is first deployed. The TSP is intended to travel and should be able to characterize 4 or 5 sites before observations begin in earnest at each site. Since these are likely to be sites where radio astronomy is conducted, a bit of useful spinoff will accrue to these observatories. 


\section{ACKNOWLEDGEMENTS}

The author wishes to thank Barney Oliver, Mike Davis, Peter Backus and her other SETI colleagues for their contributions to the design of the evolving RFI systems and for some of the graphics reproduced within this paper.

\section{REFERENCES}

Basler, R. P., Johnson, G. L. and Vondrak, R. R. "Parametric Study of Interstellar Search Systems," NASA contract report NAS2-8938, August 1976.

Duluk, J. F., Linscott, I. R., Peterson, A. M., Burr, J., Ekroot, B. and Twicken, J. "VLSI Processors for Signal Detection in SETI," paper IAA-86-489 presented at 1986 IAF Congress in Innsbruck, Austria.

Linscott, I. R., Chen, K. C. and Peterson, A. M. "The Multi-Channel Analyzer," paper presented at 1985 International Union of Radio Science Symposium, Vancouver, Canada.

Thompson, R. A. "The Response of a Radio Astronomy Synthesis Array to Interfering Signals," IEEE Transactions on Antennas and Propagation, 1982 AP-30, pp.450-456.

Welch, W. J. "The Filter Characteristics of a Multiplying Interferometer," paper LAA-86-486 presented at the 1986 LAF Congress in Innsbruck, Austria.

\section{AUTOMATED SETI SYSTEM}

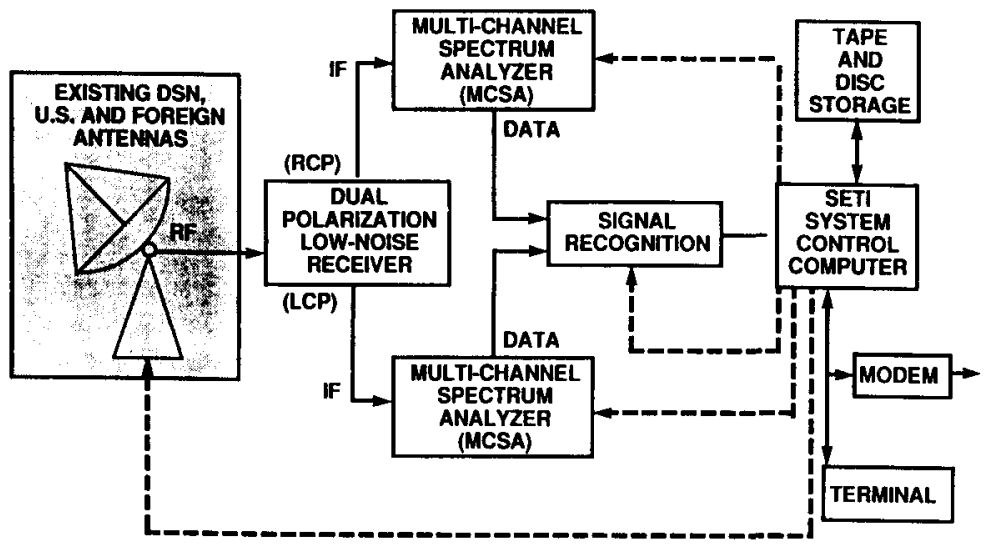

Flgure 1 - The Baceline SETI Syatem 


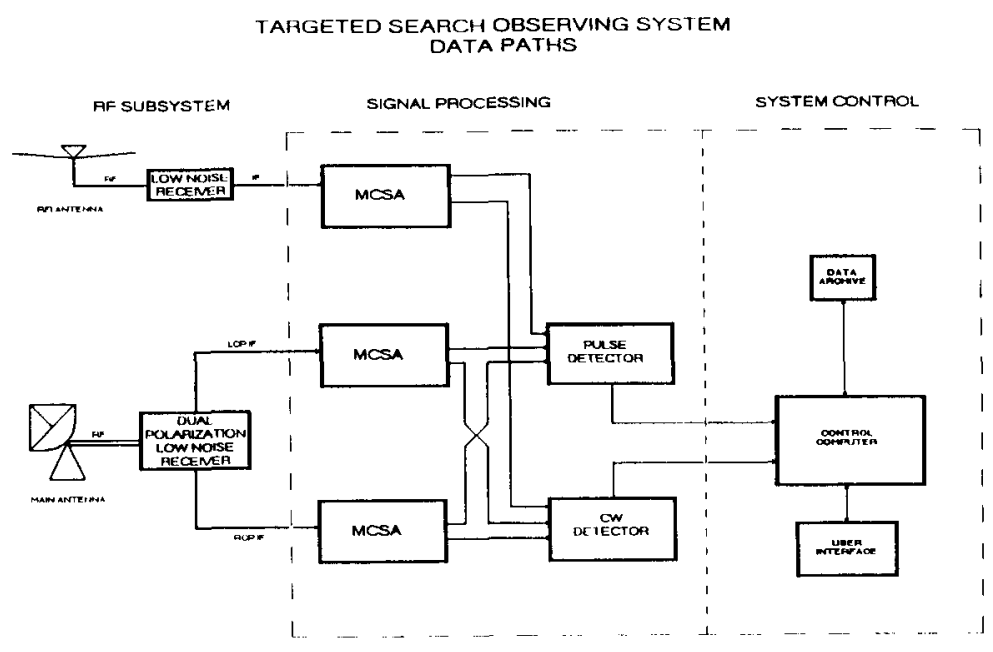

Figure 2 - Baseline + RFI Subsystem

Figure 3 - Logic for Combining Signals

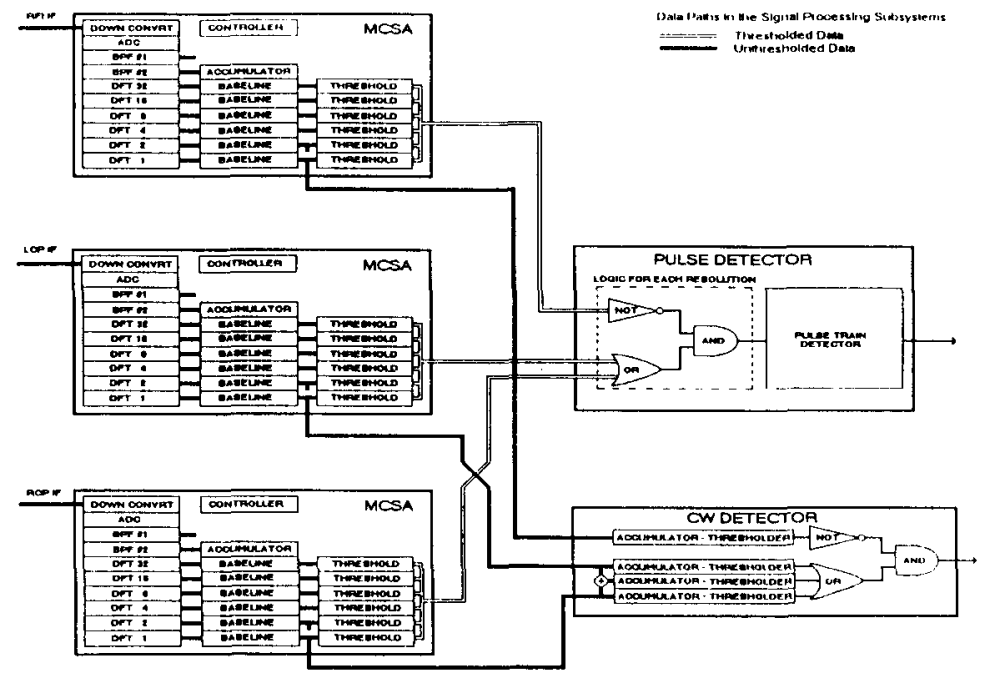

SIGNAL PROCESSING SUBSYSTEMS

OF THE TARGETED SEARCH OBSERVING SYSTEM 


\section{TARGETED SEARCH OBSERVING SYSTEM DATA PATHS}

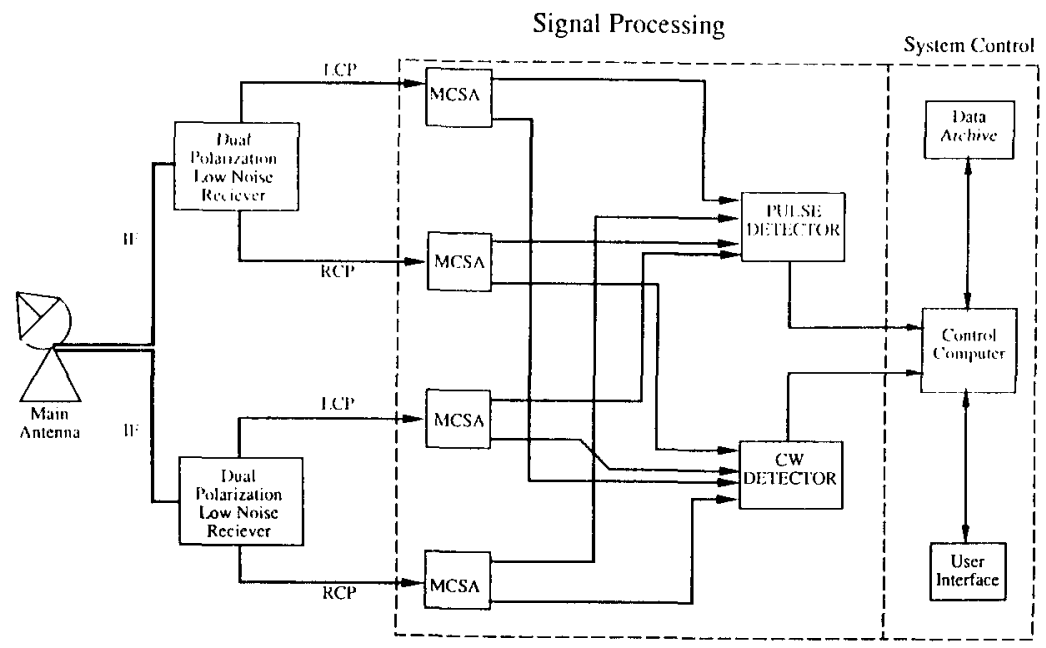

Figure 4 - Dual Beam SETI System.

\section{TARGETED SEARCH OBSERVING SYSTEM DATA PATHS}

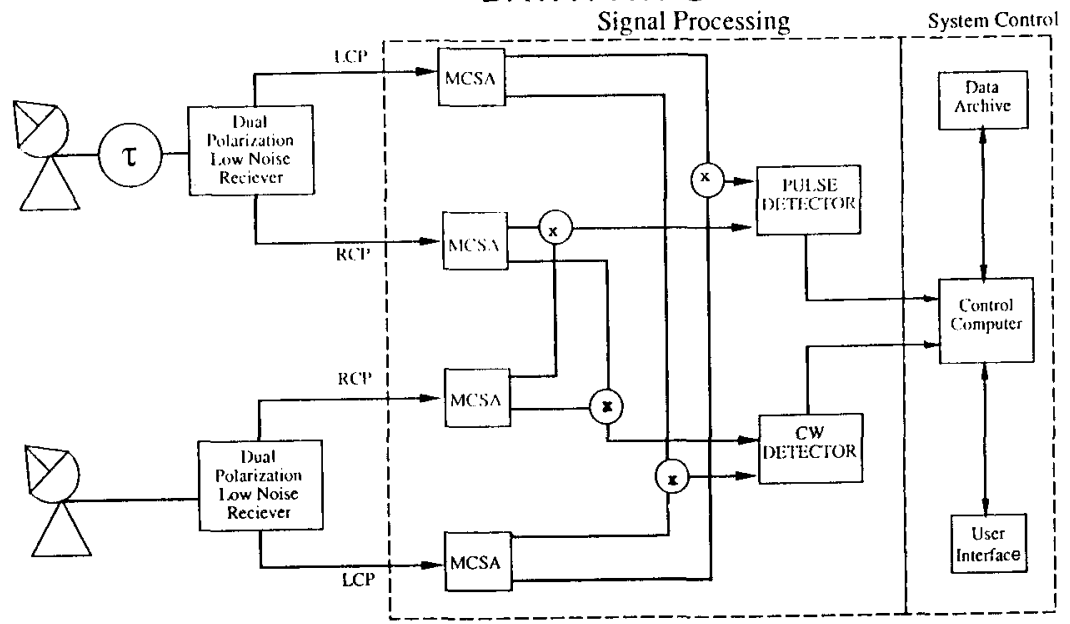

Figure S - Multiplying Interferometer 


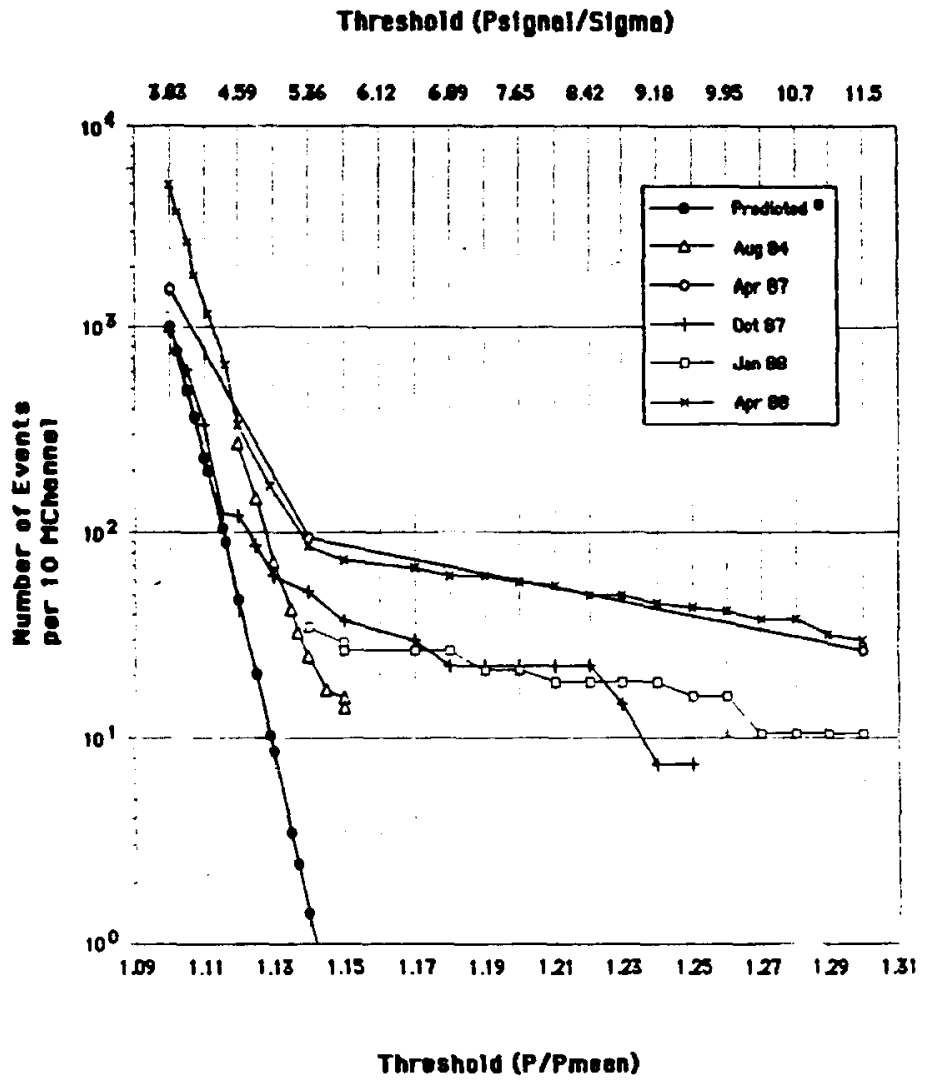

Figure 6 - Noise Statistics from Nancay Observatory 
EDM SYSTEM - MASSCOMP S60QHardware

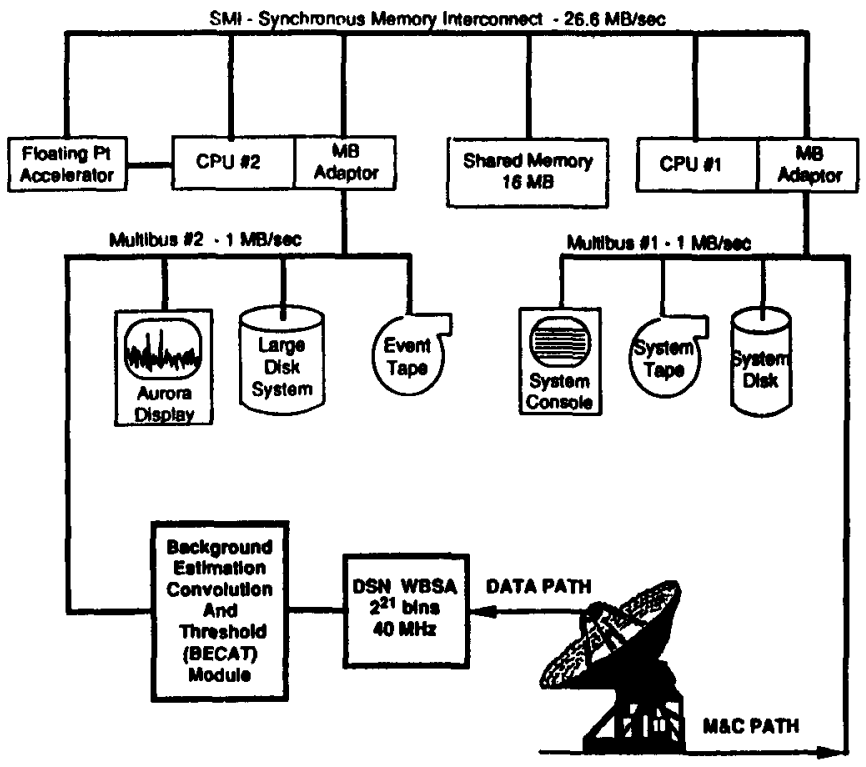

Figure 7 - Sky Survey EDM

TARGETED SEARCH PROTOTYPE (TSP)

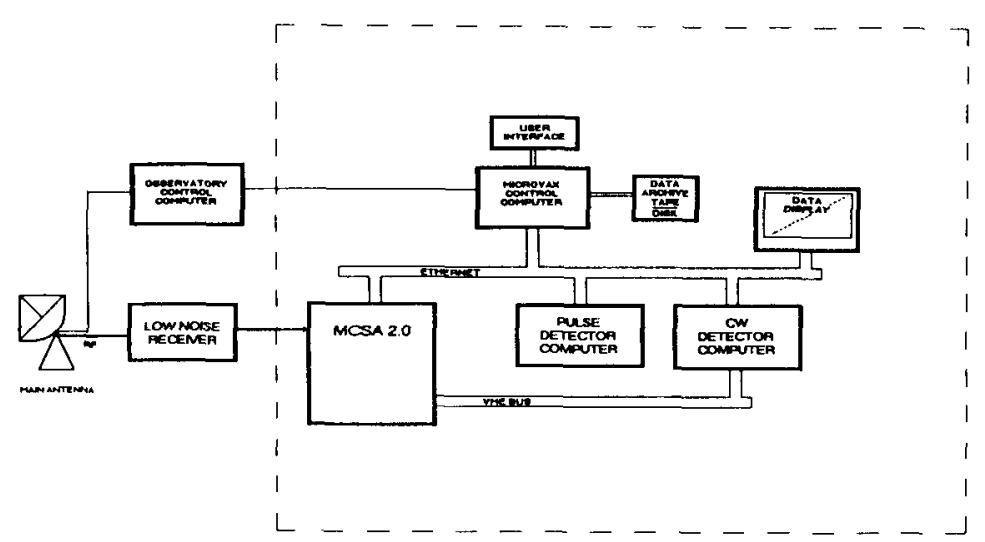

Figure 8 - The TSP 